\title{
Dislocations and dislocation reactions in decagonal Al-Ni-Co quasicrystals
}

\author{
P. Schall, ${ }^{1,2}$ M. Feuerbacher, ${ }^{2}$ and K. Urban ${ }^{2}$ \\ ${ }^{1}$ DEAS, Harvard University, Cambridge, Massachusetts 02138, USA \\ ${ }^{2}$ Institut für Festkörperforschung, Forschungszentrum Jülich, D-52425 Jülich, Germany
}

(Received 29 December 2003; published 15 April 2004)

\begin{abstract}
Dislocations in decagonal $\mathrm{Al}_{70} \mathrm{Ni}_{15} \mathrm{Co}_{15}$ and $\mathrm{Al}_{73} \mathrm{Ni}_{10} \mathrm{Co}_{17}$ were studied by means of diffraction-contrast imaging and defocus convergent beam electron diffraction techniques in the transmission electron microscope. In addition to dislocations with Burgers vectors parallel to the periodic and quasiperiodic directions as first reported by Zhang and Urban [Z. Zhang and K. Urban, Philos. Mag. Lett. 60, 97 (1989)], a type of dislocation was found possessing a Burgers vector with components in the periodic as well as the quasiperiodic direction. The parallel-space component of this Burgers vector has a length of $2.5 \AA$. The quasiperiodic Burgers vectors were found to have parallel-space lengths of 2.9 and $4.7 \AA$. The Burgers vectors determined are correlated with interatomic vectors of the structure of decagonal Al-Ni-Co. In addition, dislocation reactions were identified by the determination of Burgers vectors of dislocations in triple-node arrangements. A model for the dislocation reaction is proposed which is in accordance with the results of the microstructural studies.
\end{abstract}

DOI: 10.1103/PhysRevB.69.134105 PACS number(s): 61.14.Lj, 61.72.Dd, 61.72.Ff, 61.72.Yx

\section{INTRODUCTION}

Despite the lack of translational symmetry in quasicrystals it was shown that their plastic deformation behavior is mediated by a dislocation mechanism. ${ }^{1}$ Extensive studies were first carried out on icosahedral quasicrystalline phases. Investigations of the macroscopic deformation behavior ${ }^{2-4}$ as well as on dislocations and dislocation arrangements in differently pretreated and deformed samples ${ }^{4-6}$ have elucidated the mechanism of plastic deformation in these materials. In recent years, the class of decagonal quasicrystals has attracted increasing interest. These materials exhibit quasiperiodic order along two spatial directions and periodic order along the third. In the first plastic deformation experiments on decagonal single quasicrystals distinct anisotropies were observed. $^{7-10}$

The decagonal quasilattice may be described as a projection from a five-dimensional periodic lattice. ${ }^{11-13}$ The fivedimensional space consists of two orthogonal subspaces, the three-dimensional physical or parallel space and the twodimensional external or perpendicular space. All physicalspace reciprocal lattice vectors can be written as a linear combination of five basis vectors. However, six-dimensional basis vector systems for decagonal quasicrystals have been proposed as well. ${ }^{14,15}$ In these systems the perpendicular space is three-dimensional.

The first investigations on dislocations in decagonal quasicrystals were performed by Zhang and Urban. ${ }^{16}$ They observed two types of dislocation, with a Burgers vector along the periodic direction and with a Burgers vector in the quasiperiodic plane. The dislocations were analyzed by conventional contrast extinction experiments. Further studies by means of contrast extinction were performed by several authors. ${ }^{17-21}$ In these investigations, the same types of dislocation as reported by Zhang and Urban ${ }^{16}$ were found. The direction of the quasiperiodic Burgers vector was determined to be parallel to $P 2^{18,19}$ or to $D 2$ twofold directions ${ }^{19}$ (in the notation of Fung et $_{\text {al. }}{ }^{22}$ ).

Along with these contrast-extinction experiments, the convergent beam electron diffraction (CBED) technique was applied for studies on dislocations in decagonal quasicrystals. ${ }^{23,24}$ With this technique not only the direction but also the length of the Burgers vector can be determined. The strain field region of the dislocation is illuminated by a convergent electron beam focused above or below the sample. If $\mathbf{g} \cdot \mathbf{b} \neq 0$, where $\mathbf{b}$ is the Burgers vector of the dislocation and $\mathbf{g}$ a reciprocal-lattice vector, the corresponding higher-order Laue zone (HOLZ) line splits into $n+1$ lines forming $n$ nodes with $n=|\mathbf{g} \cdot \mathbf{b}|{ }^{25}$ The sign of $n$ can be deduced from the bending direction of the split HOLZ line. If $\mathbf{u}$ denotes the line direction of the dislocation and $\mathbf{c}$ the vector from the sample to the crossover of the electron beam, then $n$ is positive if the HOLZ line pointed at by $\mathbf{u} \times \mathbf{c}$ shifts in the direction of the diffraction vector $\mathbf{g}$, and negative if it shifts to the opposite direction. The Burgers vector $\mathbf{b}$ is then obtained by solving a set of linear equations $\mathbf{g}_{i} \cdot \mathbf{b}=n_{i}$ for linear independent diffraction vectors $\mathbf{g}_{i}$ and corresponding numbers of splitting nodes $n_{i}$.

The CBED technique, originally developed for crystalline materials, was extended to quasicrystals by Wang and Chen ${ }^{26}$ and Feng and Wang. ${ }^{27}$ The linear equation set then reads $\mathbf{G}_{i} \cdot \mathbf{B}=n_{i}$, where $\mathbf{G}_{i}$ and $\mathbf{B}$ are the higher-dimensional reciprocal-lattice and Burgers vectors, respectively. For decagonal quasicrystals five linear independent equations are needed for the determination of a Burgers vector.

HOLZ line patterns in decagonal quasicrystals have been studied experimentally and using computer simulations. ${ }^{28}$ The six-dimensional indexing system of Yan et al. ${ }^{14}$ for decagonal Al-Ni-Co was used for the simulations. The CBED technique was applied to the study of dislocations with Burgers vectors in the periodic direction ${ }^{23}$ as well as along quasiperiodic directions. ${ }^{24}$ In these publications, the Burgers vectors were expressed in the six-dimensional indexing system whereas structure models for decagonal quasicrystals are formulated based on five-dimensional systems, so that the relation between the Burgers vectors and the structure of the decagonal quasicrystal has not been established to date.

This paper presents a detailed study of dislocations in 

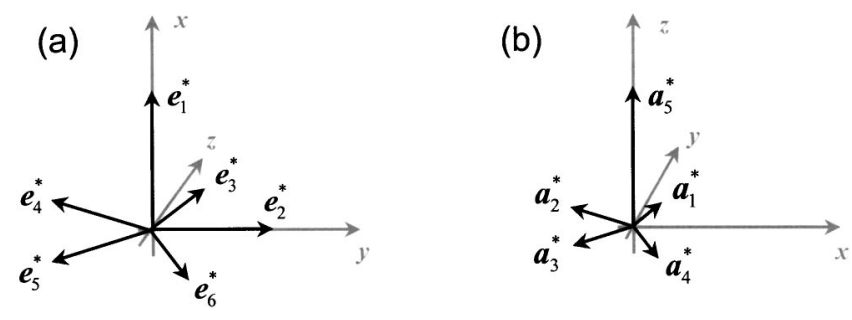

FIG. 1. Physical-space components of the six-dimensional reciprocal basis vectors of the system of Yan et al. (Ref. 14) (a) and of the five-dimensional reciprocal basis vectors of the system of Steurer et al. (Ref. 13) (b). The reciprocal basis vectors of the system of Yan et al. (Ref. 14) are larger than those of Steurer et al. (Ref. 13) by a factor of $\tau$.

decagonal Al-Ni-Co. The dislocations were analyzed by contrast-extinction experiments as well as by the defocused CBED technique. Besides dislocations of the known types, with Burgers vectors along the periodic and quasiperiodic directions, we report a type of mixed dislocation with a Burgers vector possessing components in periodic and quasiperiodic directions. The three types of Burgers vectors observed are determined both in the five- and six-dimensional notation and identified as interatomic vectors in the structure of decagonal Al-Ni-Co. We furthermore analyze dislocation arrangements and identify a specific triple-node junction, which is shown to be the product of a dislocation reaction. On the basis of the microstructural analysis we propose a model of the dislocation reaction, which, in a forthcoming paper, ${ }^{29}$ will be shown to determine the macroscopic plastic behavior of decagonal Al-Ni-Co.

\section{INDEXING SYSTEMS}

Two different indexing systems will be used throughout this paper: the six-dimensional system proposed by Yan et $a{ }^{14}$ and the five-dimensional system proposed by Steurer et al. ${ }^{13}$ The former system was applied for the simulation and indexing of HOLZ line patterns used in the CBED technique for the determination of Burgers vectors. In terms of the system of Steurer et al. ${ }^{13}$ which is widely used in the literature, various structure models of decagonal quasicrystals were formulated. ${ }^{13,30-32}$

\section{A. Six-dimensional indexing system}

In the system of Yan et al. ${ }^{14}$ the reciprocal lattice vectors of the decagonal reciprocal lattice are described by a linear combination of six basis vectors of the forms

$$
\begin{gathered}
\mathbf{e}_{1}^{*}=c^{*}(1,0,0), \\
\mathbf{e}_{i+2}^{*}=a^{*}\left[0, \cos \left(\frac{2 \pi i}{5}\right), \sin \left(\frac{2 \pi i}{5}\right)\right], \quad i=0, \ldots, 4,
\end{gathered}
$$

with the reciprocal lattice constants $c^{*}$ and $a^{*}$ in the periodic and quasiperiodic direction, respectively. These vectors are illustrated in Fig. 1(a). They are the projections of the six-dimensional basis vectors

$$
\mathbf{d}_{1}^{*}=c^{*}(1,0,0,0,0,0)
$$

and

$$
\begin{aligned}
\mathbf{d}_{i+2}^{*}= & a^{*}\left[0, \cos \left(\frac{2 \pi i}{5}\right), \sin \left(\frac{2 \pi i}{5}\right),\right. \\
& \left.\cos \left(\frac{4 \pi i}{5}\right), \sin \left(\frac{4 \pi i}{5}\right),\left(\frac{1}{2}\right)^{1 / 2}\right], \quad i=0, \ldots, 4 .
\end{aligned}
$$

The direct basis vectors spanning the unit cell in sixdimensional space can be written as

$$
\mathbf{d}_{1}=c(1,0,0,0,0,0,)
$$

and

$$
\begin{aligned}
\mathbf{d}_{i+2}= & \frac{2}{5} a\left[0, \cos \left(\frac{2 \pi i}{5}\right), \sin \left(\frac{2 \pi i}{5}\right),\right. \\
& \left.\cos \left(\frac{4 \pi i}{5}\right), \sin \left(\frac{4 \pi i}{5}\right),\left(\frac{1}{2}\right)^{1 / 2}\right], \quad i=0, \ldots, 4,
\end{aligned}
$$

where $c=1 / c^{*}$ and $a=1 / a^{*}$. Yan et al. ${ }^{14}$ determined the reciprocal lattice constants from electron diffraction patterns. For the resulting lattice constants of the direct lattice they obtain the values $2 / 5 a=0.98 \AA$ and $c=4.0 \AA$. The same reflection positions, however, can be described by quasilattice constants smaller or larger by a factor of $\tau=1 / 2(1+\sqrt{5})$, the golden mean, corresponding to a deflation or an inflation of the basis vector system. In the following, the subscript "Yan" will be used for indices which refer to the system of

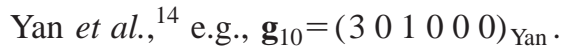

\section{B. Five-dimensional indexing system}

The indexing system of Steurer et al. ${ }^{13}$ uses the five reciprocal lattice vectors

$$
\mathbf{a}_{i}^{*}=a_{i}^{*}\left[\cos \left(\frac{2 \pi i}{5}\right), \sin \left(\frac{2 \pi i}{5}\right), 0\right], \quad i=1, \ldots 4
$$

and

$$
\mathbf{a}_{5}^{*}=a_{5}^{*}(0,0,1),
$$

with the reciprocal lattice constants $a_{i}^{*}$ and $a_{5}^{*}$ in the quasiperiodic and periodic direction, respectively. These vectors are shown in Fig. 1(b). They are the projections of the fivedimensional basis vectors

$$
\begin{aligned}
\mathbf{d}_{i}^{*}= & a_{i}^{*}\left[\cos \left(\frac{2 \pi i}{5}\right), \sin \left(\frac{2 \pi i}{5}\right), 0, \cos \left(\frac{6 \pi i}{5}\right), \sin \left(\frac{6 \pi i}{5}\right)\right] \\
i=1, \ldots, 4 &
\end{aligned}
$$

and

$$
\mathbf{d}_{5}^{*}=a_{5}^{*}(0,0,1,0,0)
$$

The direct basis vectors spanning the unit cell in fivedimensional space can be written as 


$$
\begin{aligned}
\mathbf{d}_{i}= & \frac{2}{5} a_{i}\left[\cos \left(\frac{2 \pi i}{5}\right)-1, \sin \left(\frac{2 \pi i}{5}\right), 0, \cos \left(\frac{6 \pi i}{5}\right)-1,\right. \\
& \left.\sin \left(\frac{6 \pi i}{5}\right)\right], \quad i=1, \ldots, 4
\end{aligned}
$$

and

$$
\mathbf{d}_{5}=a_{5}(0,0,1,0,0),
$$

with $a_{i}=1 / a_{i}^{*}$ and $a_{5}=1 / a_{5}^{*}$. The physical space component of the vector $\mathbf{d}_{5}$ is parallel to the periodic direction and those of the vectors $\mathbf{d}_{i}$ span the quasiperiodic plane. In the structure analysis by single-crystal $\mathrm{x}$-ray diffraction of a decagonal $\mathrm{Al}_{70} \mathrm{Ni}_{15} \mathrm{Co}_{15}$ quasicrystal Steurer et al. determined the values $a_{i}=3.794 \AA$ and $a_{5}=4.0807 \AA .{ }^{13}$ The quasiperiodic lattice constant $2 / 5 a_{i}=1.5176 \AA$ is larger than that of the indexing system of Yan et al. ${ }^{14}$ by a factor of about $\tau$. The two indexing systems thus differ not only by the number of basis vectors used for indexing but also by a scaling transformation in the quasiperiodic plane. In this publication, the subscript "Steurer" will be used for indices referring to the system of Steurer et al., ${ }^{13}$ e.g., $\mathbf{g}_{10}=\left(\begin{array}{lll}0 & 0 & -1-13\end{array}\right)_{\text {Steurer }}$.

In the structure model of Steurer et al. ${ }^{13}$ the fivedimensional hyperlattice is decorated by five different atomic surfaces per unit cell. These atomic surfaces are situated on positions at each fifth along the body diagonal of the fivedimensional unit cell. An analog to this is found in the description of the two-dimensional Penrose tiling. In the fourdimensional description of the Penrose tiling, corresponding to the five-dimensional description of the decagonal quasilattice, the hyperlattice is decorated by pentagons situated on positions at each fifth along the body diagonal of the fourdimensional unit cell. As pointed out by Ishihara and Yamamoto, ${ }^{33}$ this decoration introduces a fivefold superstructure in the four-dimensional hyperlattice.

In the five-dimensional description of the Penrose tiling corresponding to the six-dimensional description of the decagonal quasilattice, no superstructure is introduced in the higher-dimensional space. The higher-dimensional lattice is decorated by one atomic surface, a rhombic icosahedron, per unit cell. ${ }^{34}$ However, in the same way as the six-dimensional description of the decagonal quasilattice this description of the Penrose tiling is not unique since it contains an additional degree of freedom.

The transition from the six- to five-dimensional indexing system of the decagonal quasilattice can be performed as follows: Consider a six-dimensional vector with the indices $v_{1}, \ldots, v_{6}$. Introduce a basis vector system in the sixdimensional space spanning three subspaces: the threedimensional physical space $V_{\|}$, the two-dimensional perpendicular space $V_{\perp}^{(1)}$, and the additional one-dimensional perpendicular space $V_{\perp}^{(2)}$. The former two subspaces represent the five-dimensional space of the five-dimensional notation. A vector $\mathbf{v}$ may then be written as

$$
\mathbf{v}=\mathbf{v}_{\|}+\mathbf{v}_{\perp}^{(1)}+\mathbf{v}_{\perp}^{(2)},
$$

where $\mathbf{v}_{\perp}^{(2)}$ is related to the original six-dimensional indices $v_{1}, \ldots, v_{6}$ by (see, e.g., Ishihara and Yamamoto ${ }^{33}$ )

$$
\mathbf{v}_{\perp}^{(2)}=\sum_{i=2}^{6} v_{i} .
$$

We see that Eq. (7) reduces to the five-dimensional notation if the sum of the five quasiperiodic indices $v_{2}$ to $v_{6}$ of the six-dimensional description vanishes.

\section{MATERIALS}

For the microstructural investigations decagonal single quasicrystals of the compositions $\mathrm{Al}_{70.2} \mathrm{Ni}_{15.1} \mathrm{Co}_{14.7}$ and $\mathrm{Al}_{72.9} \mathrm{Ni}_{10.4} \mathrm{Co}_{16.7}$, grown by the Bridgman and the fluxgrowth technique, respectively, were used. For simplicity the two phases will be referred to as $\mathrm{Al}_{70} \mathrm{Ni}_{15} \mathrm{Co}_{15}$ and $\mathrm{Al}_{73} \mathrm{Ni}_{10} \mathrm{Co}_{17}$ in the following. The electron diffraction patterns of these quasicrystals show characteristic features of the type I superstructure and the basic cobalt phase (in the notation of Ritsch et $a l .{ }^{35}$ ), respectively. Further details on the growth and characterization are presented in Refs. 9 and 10 .

The microstructure of the quasicrystals was studied in the as-grown state as well as after uniaxial deformation to strains of about $0.5 \%$ at temperatures in the ductile temperature range. Thin foils of the material were prepared for transmission electron microscopy by mechanical grinding followed by electrochemical etching. The investigations were carried out in a JEOL 4000 FX electron microscope operated at 200 $\mathrm{kV}$.

\section{EXPERIMENTAL DETERMINATION OF THE BURGERS VECTORS IN DECAGONAL AL-NI-CO}

Figure 2 shows bright-field electron micrographs of dislocations in as-grown decagonal Al-Ni-Co under two-beam conditions with a diffraction vector parallel (left column) and perpendicular to the tenfold axis (right column). The bright field images taken with a diffraction vector perpendicular to the tenfold axis, i.e., along one of the quasiperiodic directions, show a pronounced fringe contrast.

Each row of Fig. 2 shows a different type of dislocation. The contrast of the dislocation in Figs. 2(a) and 2(b) is extinct using a diffraction vector along a quasiperiodic direction [Fig. 2(b)]. Using other quasiperiodic diffraction vectors, the dislocation contrast vanishes as well. Hence, the Burgers vector of the dislocation is parallel to the periodic axis. In our studies on decagonal $\mathrm{Al}_{70} \mathrm{Ni}_{15} \mathrm{Co}_{15}$ and $\mathrm{Al}_{73} \mathrm{Ni}_{10} \mathrm{Co}_{17}$ dislocations of this type were observed with line directions predominantly parallel or perpendicular to the tenfold axis as seen in Fig. 2(a). Figure 3(a) shows a defocused CBED pattern taken at the upper part of the dislocation with the incident beam close to a $D 2$ twofold direction. The HOLZ line corresponding to the diffraction vector $\mathbf{g}_{4 p}$

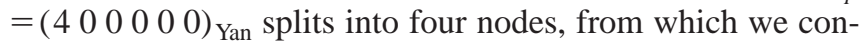
clude that the Burgers vector is of the length of one periodic lattice constant. For the CBED patterns in Fig. 3, the crossover of the incident electron beam lies below the foil. Taking the line direction $\mathbf{u}$ of the dislocation as indicated by the arrow in Fig. 3(a) we obtain the Burgers vector $\mathbf{B}^{(1)}$ $=\left[\begin{array}{llllll}-1 & 0 & 0 & 0 & 0 & 0\end{array}\right]_{\text {Yan }}=\left[\begin{array}{lllll}0 & 0 & 0 & 0 & -1\end{array}\right]_{\text {Steurer }}$. Throughout the 

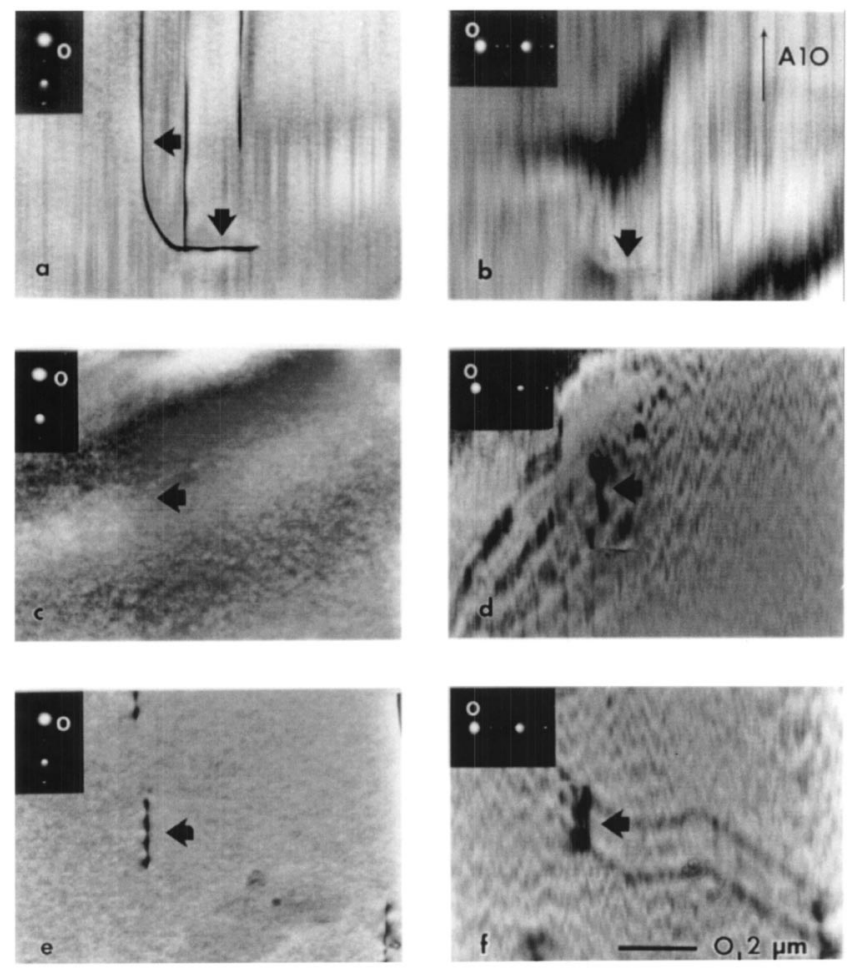

FIG. 2. Bright field images of dislocations with a Burgers vector along the periodic direction [(a) and (b)] along a quasiperiodic direction [(c) and (d)] and with a periodic and quasiperiodic component [(e) and (f)]. Diffraction vectors parallel to the periodic (left column) and a quasiperiodic direction (right column) were used for imaging.

present study Burgers vectors parallel to the periodic axis were always observed to posses a length of one lattice constant.

The contrast of the dislocation of Figs. 2(c) and 2(d) van-
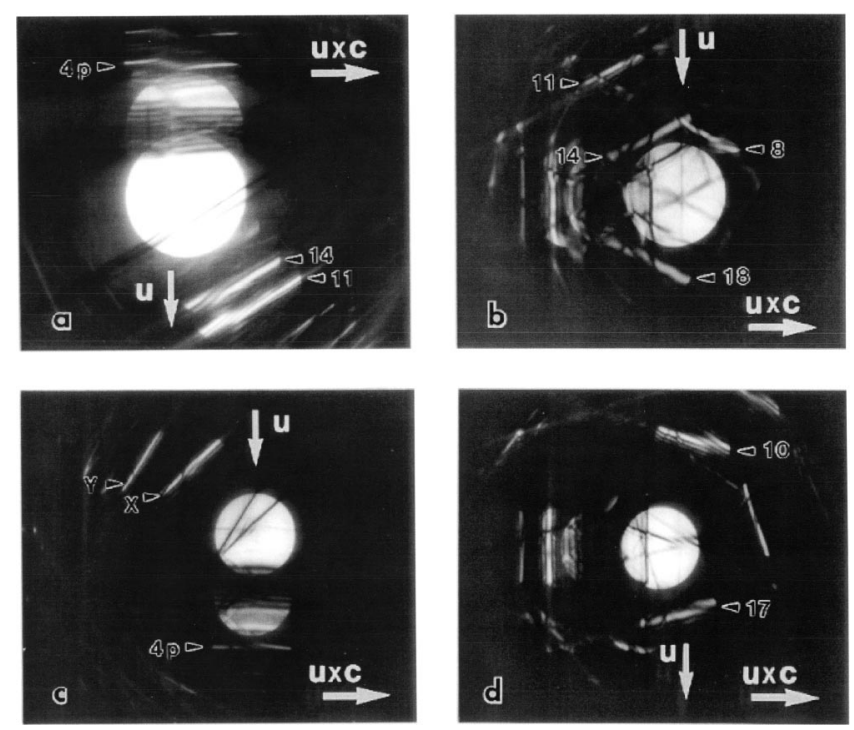

FIG. 3. Defocused CBED pattern of the dislocations with the periodic (a), the quasiperiodic (b), and the mixed Burgers vector [(c) and (d)] of Fig. 2.
TABLE I. Results of the CBED analysis of the dislocation of Figs. 2(c) and 2(d). The indices of the reciprocal lattice vectors $\mathbf{G}$ $=\mathbf{g}_{i}$ in the six- and five-dimensional notation as well as the values of the dot product $\mathbf{G}_{i} \cdot \mathbf{B}$ are shown.

\begin{tabular}{lllr}
\hline \hline $\mathbf{G}$ & \multicolumn{1}{c}{$6 D$ indices } & \multicolumn{1}{c}{$5 D$ indices } & $\mathbf{G} \cdot \mathbf{B}^{(2)}$ \\
\hline $\mathbf{g}_{8}$ & $(3,0,0,0,-1,0)$ & $(0,-1,-1,-1,3)$ & -1 \\
$\mathbf{g}_{18}$ & $(2,0,0,-1,0,-1)$ & $(0,0,-1,0,2)$ & 0 \\
$\mathbf{g}_{11}$ & $(3,-1,0,0,0,0)$ & $(0,1,1,0,3)$ & 0 \\
$\mathbf{g}_{14}$ & $(2,0,-1,0,0,-1)$ & $(1,1,1,1,2)$ & 0 \\
$\mathbf{g}_{7}$ & $(3,0,0,1,0,0)$ & $(1,1,1,0,3)$ & -1 \\
$\mathbf{g}_{10}$ & $(3,0,1,0,0,0)$ & $(0,0,-1,-1,3)$ & -1 \\
$\mathbf{g}_{9}$ & $(3,0,0,0,0,-1)$ & $(1,1,0,0,3)$ & -1 \\
\hline \hline
\end{tabular}

ishes if a diffraction vector along the periodic direction is used [Fig. 2(c)]. The Burgers vector of this dislocation thus lies in the quasiperiodic plane. Figure 3(b) shows a defocused CBED pattern of the dislocation with the incident electron beam close to a zone axis labeled " $I$ " by Yan et al. ${ }^{14}$ The HOLZ line corresponding to the diffraction vector $\mathbf{g}_{8}$ splits into one node, whereas in the HOLZ lines corresponding to the vectors $\mathbf{g}_{11}, \mathbf{g}_{14}$, and $\mathbf{g}_{18}$ no splitting occurs. The splitting behavior of the HOLZ lines of the diffraction vectors $\mathbf{g}_{7}, \mathbf{g}_{9}$, and $\mathbf{g}_{10}$ was studied as well. The results are listed in Table I together with the indices of the reciprocal lattice vectors in the six- and five-dimensional notation. Solving the linear equation set $\mathbf{G}_{i} \cdot \mathbf{B}=n_{i}$ with the reciprocal lattice vectors in the five and six-dimensional representation yields the Burgers vector

$$
\mathbf{B}^{(2)}=\left[\begin{array}{llllll}
0 & 0 & -1 & -1 & 1 & 1
\end{array}\right]_{\text {Yan }}
$$

and

$$
\mathbf{B}^{(2)}=\left[\begin{array}{lllll}
-1 & 0 & 0 & 1 & 0
\end{array}\right]_{\text {Steurer }},
$$

respectively. Note that the sum of the quasiperiodic indices in the six-dimensional notation vanishes. Hence, the sixdimensional vector directly reduces to a five-dimensional one. In order to obtain the representation (9b) referring to the system of Steurer et al. $;^{13}$ however, an additional inflation step has to be performed (see, e.g., Yan et al. ${ }^{14}$ ).

The parallel-space component $\left|\mathbf{b}_{\|}\right|$of this Burgers vector amounts to about $2.9 \AA$ and its perpendicular component $\left|\mathbf{b}_{\perp}\right|$ to $1.8 \AA$. The strain-accommodation parameter ${ }^{4} \zeta$ $=\left|\mathbf{b}_{\perp}\right| /\left|\mathbf{b}_{\|}\right|$of the dislocation amounts to $\tau^{-1}$.

Another dislocation showing the same contrast extinction behavior as that in Figs. 2(c) and 2(d) was studied by means of the defocused CBED technique. The results of the analysis are listed in Table II. Solving the linear equation set with the reciprocal lattice vectors in the five and six-dimensional representation we obtain the Burgers vectors

$$
\mathbf{B}^{(3)}=\left[\begin{array}{llllll}
0 & 1 & -1 & -2 & 0 & 2
\end{array}\right]_{\text {Yan }}
$$

and

$$
\mathbf{B}^{(3)}=\left[\begin{array}{lllll}
-1 & -1 & 0 & 1 & 0
\end{array}\right]_{\text {Steurer }} .
$$


TABLE II. Results of the CBED analysis of a dislocation showing the same contrast extinction behavior as that of Figs. 2(c) and 2(d).

\begin{tabular}{lllr}
\hline \hline $\mathbf{G}$ & \multicolumn{1}{c}{$6 D$ indices } & \multicolumn{1}{c}{$5 D$ indices } & $\mathbf{G} \cdot \mathbf{B}^{(3)}$ \\
\hline $\mathbf{g}_{8}$ & $(3,0,0,0,-1,0)$ & $(0,-1,-1,-1,3)$ & 0 \\
$\mathbf{g}_{18}$ & $(2,0,0,-1,0,-1)$ & $(0,0,-1,0,2)$ & 0 \\
$\mathbf{g}_{11}$ & $(3,-1,0,0,0,0)$ & $(0,1,1,0,3)$ & -1 \\
$\mathbf{g}_{14}$ & $(2,0,-1,0,0,-1)$ & $(1,1,1,1,2)$ & -1 \\
$\mathbf{g}_{7}$ & $(3,0,0,1,0,0)$ & $(1,1,1,0,3)$ & -2 \\
$\mathbf{g}_{10}$ & $(3,0,1,0,0,0)$ & $(0,0,-1,-1,3)$ & -1 \\
$\mathbf{g}_{9}$ & $(3,0,0,0,0,-1)$ & $(1,1,0,0,3)$ & -2 \\
\hline \hline
\end{tabular}

The sum of the quasiperiodic indices of the six-dimensional vector vanishes as well enabling again a direct transformation between the two indexing systems. The parallel and perpendicular component of the Burgers vector amount to 4.7 and $1.1 \AA$, yielding a strain-accommodation parameter of $\tau^{-3}$. Note that the parallel component of this Burgers vector is larger than that of $\mathbf{B}^{(2)}$ by a factor of $\tau$.

Figures 2(e) and 2(f) show a dislocation which is in contrast for both a diffraction vector parallel and perpendicular to the periodic axis. The Burgers vector of this dislocation thus has components both in the periodic direction and along the quasiperiodic plane. Figures 3(c) and 3(d) show defocused CBED patterns of this dislocation with the incident beam close to a $P 2$ twofold direction and a zone axis labeled " $J$ " ' by Yan et al., ${ }^{14}$ respectively. The HOLZ line corresponding to the diffraction vector $\mathbf{g}_{4 p}=\left(\begin{array}{lllllll}4 & 0 & 0 & 0 & 0 & 0\end{array}\right)_{\text {Yan }}$ parallel to the periodic direction splits into two nodes [Fig. $3(\mathrm{c})$ ], from which directly follows that the periodic component of the Burgers vector amounts to half the periodic lattice constant. The splitting behavior of the other HOLZ lines depicted in Figs. 3(c) and 3(d) suggests $\mathbf{g}_{x} \cdot \mathbf{B}=\mathbf{g}_{y} \cdot \mathbf{B}=0, \mathbf{g}_{17}$ $\cdot \mathbf{B}=1$, and $\mathbf{g}_{10} \cdot \mathbf{B}=2$. Further HOLZ lines were studied. The observed splittings are listed in Table III. Trying to solve the linear equation set $\mathbf{G}_{i} \cdot \mathbf{B}=n_{i}$ with these values we find that it has no solution in the five-dimensional notation. However, the linear equation set can be solved in the six-dimensional notation which possesses an additional degree of freedom. We obtain

TABLE III. Suggested splittings $\left(\mathbf{G}_{i} \cdot \mathbf{B}^{(4)}\right)^{\prime}$ and corrected values $\left(\mathbf{G}_{i} \cdot \mathbf{B}^{(4)}\right)$ of the dislocation shown in Figs. 2(e) and 2(f) with the reciprocal lattice vectors $\mathbf{G}=\mathbf{g}_{i}$ in the six- and five-dimensional notations.

\begin{tabular}{lllcc}
\hline \hline $\mathbf{G}$ & $5 D$ indices & $6 D$ indices & $\left(\mathbf{G} \cdot \mathbf{B}^{(4)}\right)^{\prime}$ & $\mathbf{G} \cdot \mathbf{B}^{(4)}$ \\
\hline $\mathbf{g}_{x}$ & $(1,1,2,1,2)$ & $(2,0,-1,1,0,0)$ & 0 & 0 \\
$\mathbf{g}_{y}$ & $(0,2,2,1,2)$ & $(2,-1,0,0,1,0)$ & 0 & 0 \\
$\mathbf{g}_{4 p}$ & $(0,0,0,0,4)$ & $(4,0,0,0,0,0)$ & 2 & 2 \\
$\mathbf{g}_{10}$ & $(0,0,-1,-1,3)$ & $(3,0,1,0,0,0)$ & 2 & 1,9 \\
$\mathbf{g}_{17}$ & $(0,1,0,0,2)$ & $(2,0,1,0,1,0)$ & 1 & 0,8 \\
$\mathbf{g}_{8}$ & $(0,-1,-1,-1,3)$ & $(3,0,0,0,-1,0)$ & 2 & 2,1 \\
$\mathbf{g}_{11}$ & $(0,1,1,0,3)$ & $(3,-1,0,0,0,0)$ & 1 & 1,1 \\
$\mathbf{g}_{9}$ & $(1,1,0,0,3)$ & $(3,0,0,0,0,-1)$ & 1 & 1,1 \\
\hline \hline
\end{tabular}

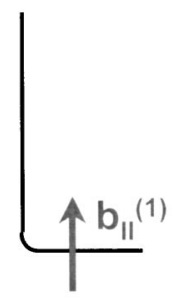

(a)

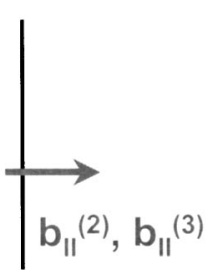

(b)

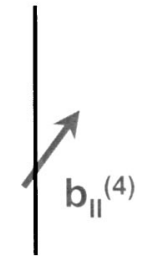

(c)
FIG. 4. Types of dislocations in decagonal Al-Ni-Co periodic (a), quasiperiodic (b), and mixed (c). The Burgers vector is illustrated by a gray arrow.

$$
\mathbf{B}^{(4)^{\prime}}=\left[\begin{array}{llllll}
\frac{1}{2} & \frac{1}{2} & \frac{1}{2} & -\frac{1}{2} & -\frac{1}{2} & \frac{1}{2}
\end{array}\right]_{\mathrm{Yan}} .
$$

The sum of the quasiperiodic indices of this vector does not vanish. However, we can correct this vector by adding a constant $c$ to all quasiperiodic indices. Owing to the degree of freedom of the six-dimensional notation this does not change the physical-space component of the vector. With $c$ $=1 / 10$ we obtain

$$
\mathbf{B}^{(4)}=\left[\begin{array}{llllll}
\frac{1}{2} & \frac{2}{5} & \frac{2}{5} & -\frac{3}{5} & -\frac{3}{5} & \frac{2}{5}
\end{array}\right]_{\mathrm{Yan}} .
$$

The dot products of this vector with the reciprocal lattice vectors are either equal or very close to the values determined from the CBED pattern (Table III). The sum of the quasiperiodic indices of the corrected Burgers vector $\mathbf{B}^{(4)}$ vanishes. The representation of $\mathbf{B}^{(4)}$ in the five-dimensional indexing system can be obtained either by direct transformation of the six-dimensional vector (12) or by solving the linear equation set with the corrected values $\mathbf{G}_{i} \cdot \mathbf{B}$, yielding the vector

$$
\mathbf{B}^{(4)}=\left[\begin{array}{lllll}
-\frac{1}{5} & -\frac{1}{5} & -\frac{1}{5} & -\frac{1}{5} & \frac{1}{2}
\end{array}\right]_{\text {Steurer }} .
$$

The parallel-space component of this vector amounts to about $2.5 \AA$.

The three different types of dislocations found in the present study are illustrated in Fig. 4. The physical-space component of the Burgers vector is shown as a gray arrow in this schematic representation. The dislocations with the Burgers vector parallel to the periodic direction (a), in the quasiperiodic plane (b), and with a periodic and quasiperiodic component (c) will be referred to as periodic, quasiperiodic, and mixed dislocations, respectively, in the following. It is important to note that the line direction of the quasiperiodic and mixed dislocations was always observed to be parallel to the periodic axis, whereas the line direction of periodic dislocations was observed to be preferentially parallel or perpendicular to the periodic axis. 


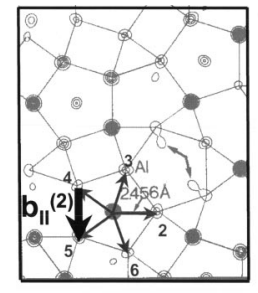

(a)

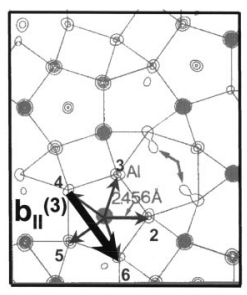

(b)

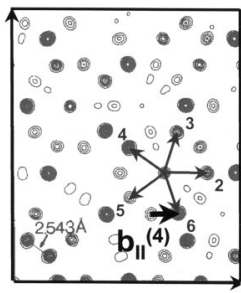

(c)
FIG. 5. Physical space components $\mathbf{b}_{\|}^{(2)}$ and $\mathbf{b}_{\|}^{(3)}$ of the two quasiperiodic Burgers vectors depicted in a section of the structure of a quasiperiodic plane after Steurer et al. ${ }^{13}[(a)$ and (b)] and physical space component $\mathbf{b}_{\|}^{(4)}$ of the mixed Burgers vector depicted in a section of the projected structure (c). The basis vectors labeled by 2 to 6 are larger than those of Steurer et al. (Ref. 13) by a factor of $\tau$.

\section{IDENTIFICATION OF THE BURGERS VECTORS IN THE STRUCTURE OF DECAGONAL AL-NI-CO}

The Burgers vectors $\mathbf{B}^{(1)}$ to $\mathbf{B}^{(4)}$ determined experimentally by the defocused CBED technique can be identified as interatomic vectors in the structure of decagonal Al-Ni-Co (Ref. 13) according to their component in physical space. For this we consider a section of the structure of the decagonal quasicrystal along the quasiperiodic plane. For best illustration, we chose a six-dimensional basis vector system with the five basis vectors in the quasiperiodic plane pointing to the vertices of a pentagonal motif of the structure [vectors 2-6 in Figs. 5(a) and 5(b)]. These basis vectors are larger than the basis vectors of the system of Steurer et al. ${ }^{13}$ by a factor of $\tau$ and larger than those of Yan et al. ${ }^{14}$ by a factor of $\tau^{2}$. The indices of the Burgers vectors $\mathbf{B}^{(1)}$ to $\mathbf{B}^{(4)}$ with respect to the basis vector system are thus obtained from the indices in the notation of Yan et al. ${ }^{14}$ by a twofold inflation operation. For the two quasiperiodic Burgers vectors we obtain the representations

$$
\mathbf{B}^{(2)}=\left[\begin{array}{llllll}
0 & 0 & 0 & -1 & 1 & 0
\end{array}\right]_{\text {Yan }}^{\prime \prime}
$$

and

$$
\mathbf{B}^{(3)}=\left[\begin{array}{llllll}
0 & 0 & 0 & -1 & 0 & 1
\end{array}\right]_{\text {Yan }}^{\prime \prime},
$$

in the two times inflated basis vector system using the inflation matrix $\tilde{\mathbf{M}}^{-1}$ given by Yan et al. ${ }^{14}$ The physical-space components of these vectors are depicted by fat arrows in Figs. 5(a) and 5(b). They correspond to vectors between nearest or second nearest vertices of the pentagonal structure motif.

The periodic component of the mixed Burgers vector amounts to half the periodic lattice constant, i.e., this vector points to the next quasiperiodic plane. For the identification of the vector we transform the quasiperiodic component to the $\tau^{2}$-times inflated basis vector system as before, and obtain

$$
\mathbf{B}^{(4)}=\left[\begin{array}{llllll}
\frac{1}{2} & -\frac{2}{5} & \frac{3}{5} & -\frac{2}{5} & -\frac{2}{5} & \frac{3}{5}
\end{array}\right]_{\mathrm{Yan}}^{\prime \prime} .
$$
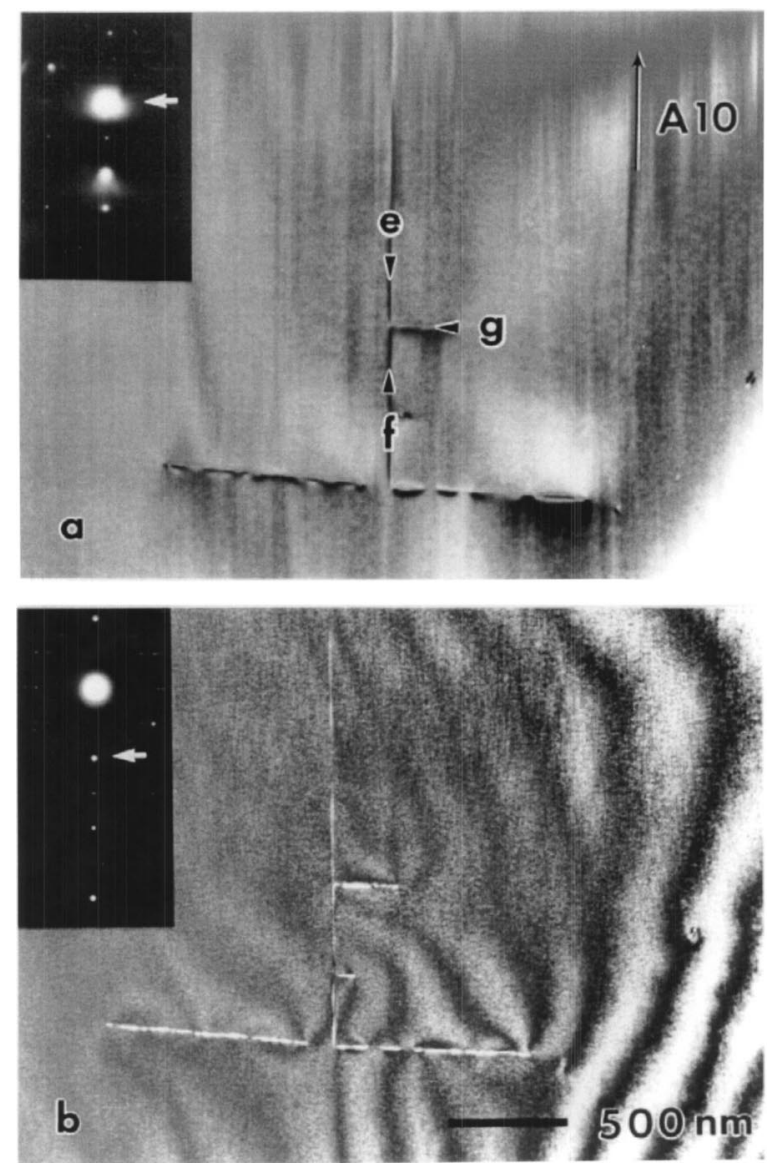

FIG. 6. Bright field (a) and weak-beam dark-field images (b) of a dislocation arrangement studied by the defocused CBED technique. The diffraction vectors are parallel to the periodic axis A10.

This vector has noninteger quasiperiodic indices. Hence its identification in the structure is not as obvious as that of the quasiperiodic Burgers vectors before. However, we can add the number $2 / 5$ to all quasiperiodic indices obtaining the vector $\left[\begin{array}{llllll}\frac{1}{2} & 0 & 1 & 0 & 0 & 1\end{array}\right]_{\text {Yan }}^{\prime \prime}$, which has the same component in physical space as $\mathbf{B}^{(4)}$. This vector can be easily identified in the structure. Figure 5(c) shows the physical space component of this vector in a section of the projected structure of decagonal Al-Ni-Co. It corresponds to a vector between two nearest atoms of neighboring quasiperiodic planes.

\section{DISLOCATION REACTIONS}

Figure 6(a) shows a bright-field electron micrograph of an arrangement of dislocations with line directions parallel and perpendicular to the tenfold axis. This dislocation arrangement was observed in a sample deformed in compression parallel to the tenfold axis. The dislocation segments marked by arrowheads labeled $e, f$, and $g$ meet at a junction. Contrast extinction studies show that the dislocation segment $g$ has a periodic Burgers vector whereas the segments $e$ and $f$ have mixed Burgers vectors. Figure 6(b) shows a weak-beam dark-field image of the dislocation arrangement. No splitting of the dislocation lines can be recognized in this image. Figures 7(a)-7(c) show defocused CBED patterns of the dislo- 

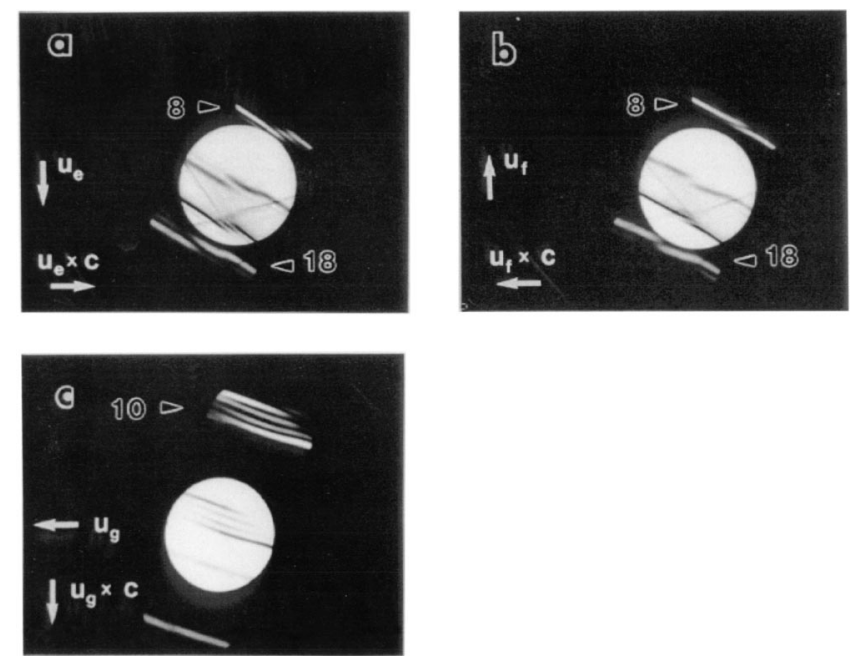

FIG. 7. Defocused CBED patterns taken from the areas containing the dislocation segments $e$ (a), $f$ (b), and $g$ (c) of Fig. 6. The HOLZ lines are labeled by numbers which correspond to the reciprocal lattice vectors listed in Table IV. The line direction $\mathbf{u}$ of the dislocation segment and the direction of the cross product $\mathbf{u} \times \mathbf{c}$ are indicated by arrows.

cation segments $e$ to $g$, respectively. For these patterns the crossover of the incident beam lies below the foil and hence the vector $c$ between the foil and the crossover points downwards, i.e., along the direction of the electron beam. The line direction $\mathbf{u}$ as defined by the arrowheads in Fig. 6(a) and the direction of the cross product $\mathbf{u} \times \mathbf{c}$ are indicated in the figures. In the CBED pattern of the periodic dislocation [Fig. 7(c)] only the HOLZ line corresponding to the diffraction vector $\mathbf{g}_{10}=\left(\begin{array}{llllll}3 & 0 & 1 & 0 & 0 & 0\end{array}\right)_{\text {Yan }}$ is affected by the dislocation segment. This HOLZ line splits into three nodes. The length of the Burgers vector thus amounts to one periodic lattice constant, in accordance with our earlier studies. The deficient line pointed at by $\mathbf{u}_{g} \times \mathbf{c}$ shifts to the direction opposite to $\mathbf{g}_{10}$, which means that the sense of the Burgers vector is along the negative $A 10$ direction, i.e., $\mathbf{B}_{g}$

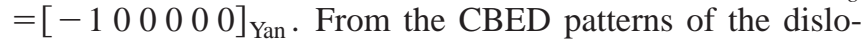
cation segments $e$ and $f$ we read the splittings of the HOLZ lines as listed in Table IV. Solving the corresponding linear equation sets we obtain the Burgers vectors $\mathbf{B}_{e}^{\prime}=1 / 2[11$

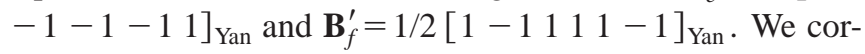
rect these vectors as described in Sec. IV, obtaining

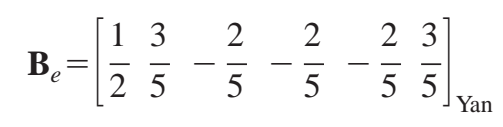

and

$$
\mathbf{B}_{f}=\left[\begin{array}{llllll}
\frac{1}{2} & -\frac{3}{5} & \frac{2}{5} & \frac{2}{5} & \frac{2}{5} & -\frac{3}{5}
\end{array}\right]_{\mathrm{Yan}} .
$$

The sum of the Burgers vectors of all three dislocation segments amounts to

$$
\mathbf{B}_{e}+\mathbf{B}_{f}+\mathbf{B}_{g}=0
$$

TABLE IV. Suggested splittings in the CBED pattern of the dislocation segments $e$ (a) and $f($ b) shown in Fig. 6.

(a)

\begin{tabular}{llc}
\hline $\mathbf{G}$ & \multicolumn{1}{c}{$6 D$ indices } & $\left(\mathbf{G} \cdot \mathbf{B}_{e}\right)^{\prime}$ \\
\hline $\mathbf{g}_{8}$ & $(3,0,0,0,-1,0)$ & 2 \\
$\mathbf{g}_{18}$ & $(2,0,0,-1,0,1)$ & 1 \\
$\mathbf{g}_{14}$ & $(2,0,-1,0,0,-1)$ & 1 \\
$\mathbf{g}_{10}$ & $(3,0,1,0,0,0)$ & 1 \\
$\mathbf{g}_{15}$ & $(2,1,0,1,0,0)$ & 1 \\
$\mathbf{g}_{11}$ & $(3,-1,0,0,0,0)$ & 1 \\
$\mathbf{g}_{7}$ & $(3,0,0,1,0,0)$ & 1
\end{tabular}

(b)

\begin{tabular}{llc}
\hline $\mathbf{G}$ & $6 D$ indices & $\left(\mathbf{G} \cdot \mathbf{B}_{f}\right)^{\prime}$ \\
\hline $\mathbf{g}_{8}$ & $(3,0,0,0,-1,0)$ & 1 \\
$\mathbf{g}_{18}$ & $(2,0,0,-1,0,1)$ & 1 \\
$\mathbf{g}_{14}$ & $(2,0,-1,0,0,-1)$ & 1 \\
$\mathbf{g}_{10}$ & $(3,0,1,0,0,0)$ & 2 \\
$\mathbf{g}_{15}$ & $(2,1,0,1,0,0)$ & 1 \\
$\mathbf{g}_{11}$ & $(3,-1,0,0,0,0)$ & 2 \\
$\mathbf{g}_{7}$ & $(3,0,0,1,0,0)$ & 2 \\
\hline \hline
\end{tabular}

indicating that the analyzed dislocation arrangement is the product of a dislocation reaction.

In order to elucidate the mechanism of the dislocation reaction a second dislocation arrangement was studied (see Fig. 8). The dislocation parallel to the tenfold axis with the segments $k$ and $h$ at the lower and the upper part of the figure, respectively, forms two junctions, one with each of the dislocation segments $l$ and $m$. As before we determine the Burgers vectors of the dislocation segments by the CBED technique, obtaining

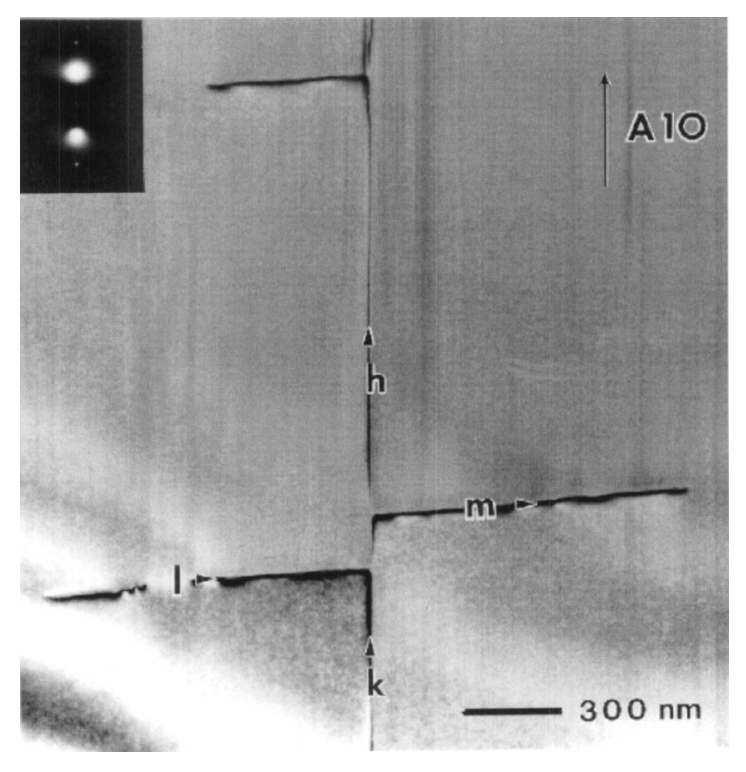

FIG. 8. Bright field image of a dislocation arrangement studied by the defocused CBED technique. The diffraction vector is parallel to the periodic axis A10. 


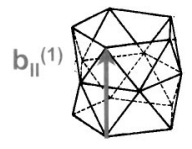

(a)

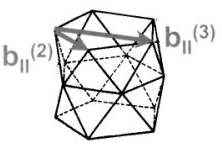

(b)

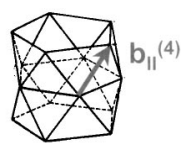

(c)
FIG. 9. Relation between the periodic (a), the quasiperiodic (b) and the mixed Burgers vector (c) and the pentagonal antiprismatic channel. The latter is an elementary structural building block of decagonal Al-Ni-Co and approximant phases.

$$
\mathbf{B}_{1}=\mathbf{B}_{m}=\left[\begin{array}{llllll}
1 & 0 & 0 & 0 & 0 & 0
\end{array}\right]_{\text {Yan }}
$$

and

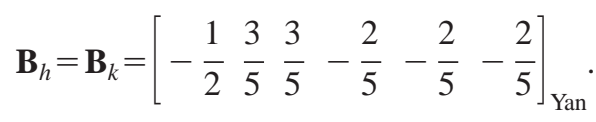

The Burgers vectors of the dislocation segments to the left and to the right, $l$ and $m$, as well as those of the segments below and above the two junctions, $k$ and $h$, are equal.

\section{DISCUSSION}

Three types of dislocations are observed in decagonal Al$\mathrm{Ni}$-Co. Dislocations with a Burgers vector along the periodic direction, with a Burgers vector in the quasiperiodic plane and with a Burgers vector possessing both a periodic and a quasiperiodic component. The Burgers vectors were explicitly determined in a five- and six-dimensional indexing system and identified in the structure of the decagonal quasicrystal according to the structure model of Steurer et al. ${ }^{13}$ The quasiperiodic Burgers vectors point to the nearest and second nearest neighbors in the quasiperiodic plane and the mixed Burgers vector points to the nearest neighbor in the next quasiperiodic plane below or above. As pointed out by Steurer et al. $^{13}$ the pentagonal structure motif in the quasiperiodic plane builds up a pentagonal antiprismatic channel in the three-dimensional structure with the pentagons of the layers above and below. This pentagonal antiprismatic channel is known as the basic structural building block of decagonal Al-Ni-Co and approximant phases. The Burgers vectors determined in this study lie either along the edges of the pentagonal antiprismatic channel or point to the second nearest vertex (Fig. 9) clearly showing that they correspond to basic vectors in the structure decagonal Al-Ni-Co.

The mixed Burgers vector has non-integer quasiperiodic indices, multiples of one-fifth, in consistence with the fivefold superstructure in the five-dimensional space. Hence, it is a partial dislocation in the higher-dimensional space. Accordingly, a stacking fault should be observed in the bright field images. As Fig. 2 shows we indeed observe a stacking fault attached to the mixed dislocation. However, we were not able to systematically extinguish the contrast of the stacking fault using specific reciprocal lattice vectors for imaging. This may be due to the inherent structural disorder of the decagonal phases studied.

Along with the Burgers vector, the dot product $\mathbf{G} \cdot \mathbf{B}$ of the mixed dislocations shows non-integer values as well, deviating from integer values by $1 / 5$ or $1 / 10$. In our CBED pattern

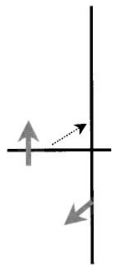

(a)

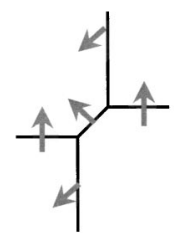

(b)

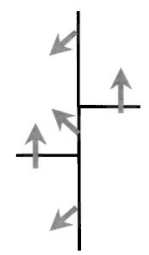

(c)
FIG. 10. Schematic of the reaction between dislocations with a periodic and a mixed Burgers vector. The Burgers vector is illustrated by a gray arrow. See the text.

recorded we were not able to distinguish deviations of this order of magnitude from the nearest integer values. This is the first time that CBED pattern of partial dislocations have been reported for quasicrystals. For crystalline materials, Tanaka et al. ${ }^{36}$ simulated defocused CBED patterns of partial dislocations. In the simulated images the deviation of the dot product from the integer value is recognized as a slight intensity shift in the HOLZ lines. However, even deviations of one third from integer numbers can hardly be distinguished from CBED patterns corresponding to integer numbers.

The quasiperiodic Burgers vectors observed possess lengths of 2.9 and $4.7 \AA$ and strain-accommodation parameters of $\tau^{-1}$ and $\tau^{-3}$. The first one corresponds to a Burgers vector previously reported. ${ }^{24}$ The ratio of the perpendicular component of the Burgers vector to the size of the atomic surface, $\left|\mathbf{b}_{\perp}\right| / A$, is proportional to the number density of phason defects associated with the dislocation. With a typical size of the atomic surface amounting to about $A=1.5 \AA$ for decagonal Al-Ni-Co, ${ }^{13}$ we obtain $\left|\mathbf{b}_{\perp}\right| / A$ ratios of about 0.7 and 1.2 for the $\tau^{-3}$ and $\tau^{-1}$ dislocation, respectively. These numbers are low compared to dislocations in icosahedral AlPd-Mn. There, Burgers vectors with strain-accommodation parameters in the range of $\tau^{3}$ to $\tau^{9}$ are reported, ${ }^{5}$ yielding $\left|\mathbf{b}_{\perp}\right| / A$ ratios ranging from 1.2 to 5.3 , taking the size of the atomic surface to be about $10 \AA{ }^{37}$ This means that dislocations in icosahedral Al-Pd-Mn show a tendency to a higher density of associated phason defects than those in decagonal Al-Ni-Co.

The line directions of all the dislocations observed have a preferential orientation parallel or perpendicular to the periodic axis. The periodic dislocations are observed with both line directions whereas the dislocations with quasiperiodic or mixed Burgers vectors are only found with line directions parallel to the periodic axis. The restriction of the line direction and the small number of different types of Burgers vectors limits the number of slip systems for plastic deformation. This has important consequences for the deformation behavior of the decagonal phase, in particular with regard to anisotropies. $^{29}$

A decisive role for the plastic behavior of decagonal quasicrystals is played by the dislocation reaction observed in the present work. ${ }^{29}$ Our Burgers vector analysis and Fig. 8 imply the following dislocation reaction (Fig. 10): a periodic and a mixed dislocation meet [Fig. 10(a)]. The components of the Burgers vectors along the periodic direction are of opposite sign. The two dislocations react forming a disloca- 
tion segment with a mixed Burgers vector with the periodic component in the opposite direction [Fig. 10(b)]. Since the mixed dislocation has a preferential orientation parallel to the periodic axis, the configuration shown in Fig. 10(c) will be observed in spite of that in Fig. 10(b). This configuration exactly corresponds to that of Fig. 8 as observed in the experiment. Upon reaction, the energy of the configuration is reduced by the energy cost of the periodic dislocation. Hence, the dislocation reaction is energetically favorable.

The occurrence of other dislocation reactions in decagonal Al-Ni-Co is very limited. Provided that the product of the dislocation reaction is still a dislocation of one of the three types observed, only very few dislocation reactions can be imagined. The reaction between a periodic and a quasiperiodic dislocation, for example, can be ruled out because the resulting Burgers vector would be too large. Mixed dislocations possessing Burgers vectors with one lattice constant in the periodic direction were never observed in our studies. Furthermore, we can rule out the reaction between a mixed and a quasiperiodic dislocation. The quasiperiodic components of the mixed Burgers vectors are parallel to $P 2$ twofold directions, whereas that of the quasiperiodic Burgers vectors are parallel to $D 2$ twofold directions. Hence, the sum of the two would not result in another mixed Burgers vector of the type observed. Reactions between two periodic dislocations can be ruled out as well except for the case of annihilation, since the sum of two periodic Burgers vectors of the same sign would be too large. Two conceivable cases remain: The reaction of two mixed or two quasiperiodic dislocations. Two mixed dislocations may react forming a periodic dislocation if their quasiperiodic components are antiparallel and their periodic components are parallel. However, the dislocation reaction takes place only if it is energetically favorable. In crystals, the elastic energy of a dislocation is proportional to the square of its Burgers vector modulus. ${ }^{38}$ The reaction between dislocations with Burgers vectors $\mathbf{b}_{1}$ and $\mathbf{b}_{2}$ is energetically favorable if $\left(\mathbf{b}_{1}+\mathbf{b}_{2}\right)^{2}$ $<\mathbf{b}_{1}^{2}+\mathbf{b}_{2}^{2}$ (Frank's rule) meaning that the two Burgers vectors include an angle smaller than $90^{\circ}$. In quasicrystals both the parallel and perpendicular-space component of the Burgers vector has to be considered for the calculation of the dislocation energy. ${ }^{39-42}$ However, little is known about the energy contribution of the perpendicular component. Since in decagonal Al-Ni-Co the perpendicular component of $\mathbf{b}$ was found to be comparatively small, we restrict our energy consideration to the physical-space component only. For the two mixed dislocations we find that the dislocation reaction is then energetically favorable since the Burgers vectors include an angle of $73^{\circ}$. Reactions between quasiperiodic dislocations may take place in two ways.

(a) Two dislocations with a quasiperiodic Burgers vector of the type $\mathbf{B}^{(2)}$ react forming a dislocation with a Burgers vector of type $\mathbf{B}^{(3)}$, e.g.,

$$
\begin{aligned}
& {\left[\begin{array}{llllll}
0 & 0 & -1 & -1 & 1 & 1
\end{array}\right]_{\text {Yan }}+\left[\begin{array}{llllll}
0 & -1 & -1 & 1 & 1 & 0
\end{array}\right]_{\text {Yan }}} \\
& \quad \rightarrow\left[\begin{array}{llllll}
0 & -1 & -2 & 0 & 2 & 1
\end{array}\right]_{\text {Yan }} .
\end{aligned}
$$

(b) Two dislocations with a quasiperiodic Burgers vector of the type $\mathbf{B}^{(3)}$ react resulting in a dislocation with a Burgers vector of type $\mathbf{B}^{(2)}$, e.g.,

$$
\begin{aligned}
& {\left[\begin{array}{llllll}
0 & 2 & 1 & -1 & -2 & 0
\end{array}\right]_{\text {Yan }}+\left[\begin{array}{llllll}
0 & -1 & -2 & 0 & 2 & 1
\end{array}\right]_{\text {Yan }}} \\
& \quad \rightarrow\left[\begin{array}{llllll}
0 & 1 & -1 & -1 & 0 & 1
\end{array}\right]_{\text {Yan. }} .
\end{aligned}
$$

In the first case, the physical space component of the resulting Burgers vector is longer and in the second case it is shorter by a factor of $\tau$ than that of the Burgers vectors of the reacting dislocations. This means that in our approximation only the second dislocation reaction is energetically favorable. Since the line directions of two quasiperiodic dislocations are always parallel to each other, however, these reactions are expected to occur very rarely.

The line directions between reacting mixed and periodic dislocations generally are perpendicular to each other, so that the dislocation reactions are expected to occur frequently. The dislocation reaction occurs along a small part of the dislocation line of the mixed dislocation only. The remaining dislocation segments of the periodic dislocation, e.g., segments $l$ and $m$ in Fig. 8, can exhibit further reactions with other mixed dislocations. In this way, a network of periodic and mixed dislocations builds up. As will be shown in a forthcoming publication ${ }^{29}$ this has important consequences for the deformation behavior of the decagonal phase.

\section{ACKNOWLEDGMENT}

The authors are grateful to Professor R. Wang for fruitful discussions.
${ }^{1}$ M. Wollgarten, M. Beyss, K. Urban, H. Liebertz, and U. Köster, Phys. Rev. Lett. 71, 549 (1993).

${ }^{2}$ M. Feuerbacher, B. Baufeld, R. Rosenfeld, M. Bartsch, G. Hanke, M. Beyss, M. Wollgarten, U. Messerschmidt, and K. Urban, Philos. Mag. Lett. 72, 91 (1995).

${ }^{3}$ B. Geyer, M. Bartsch, M. Feuerbacher, K. Urban, and U. Messerschmidt, Philos. Mag. A 80, 1151 (2000).

${ }^{4}$ M. Feuerbacher, C. Metzmacher, M. Wollgarten, K. Urban, B. Baufeld, M. Bartsch, and U. Messerschmidt, Mater. Sci. Eng., A 233, 103 (1997).

${ }^{5}$ R. Rosenfeld, M. Feuerbacher, B. Baufeld, M. Bartsch, M. Woll- garten, G. Hanke, M. Beyss, U. Messerschmidt, and K. Urban, Philos. Mag. Lett. 72, 375 (1995).

${ }^{6}$ P. Schall, M. Feuerbacher, M. Bartsch, U. Messerschmidt, and K. Urban, Philos. Mag. Lett. 79, 785 (1999).

${ }^{7}$ M. Feuerbacher, M. Bartsch, G. Grushko, U. Messerschmidt, and K. Urban, Philos. Mag. Lett. 76, 369 (1997).

${ }^{8}$ K. Edagawa, Y. Arai, T. Hashimoto, and S. Takeuchi, Mater. Trans., JIM 39, 863 (1998).

${ }^{9}$ P. Schall, M. Feuerbacher, and K. Urban, Philos. Mag. Lett. 81, 339 (2001)

${ }^{10}$ P. Schall, M. Feuerbacher, and K. Urban (unpublished). 
${ }^{11}$ A. Yamamoto and K. N. Ishihara, Acta Crystallogr., Sect. A: Found. Crystallogr. 44, 707 (1988).

${ }^{12}$ W. Steurer, Acta Crystallogr., Sect. B: Struct. Sci. 45, 534 (1989).

${ }^{13}$ W. Steurer, T. Haibach, B. Zhang, S. Kek, and R. Lück, Acta Crystallogr., Sect. B: Struct. Sci. 49, 661 (1993).

${ }^{14}$ Y. Yan, R. Wang, J. Gui, and M. Dai, Acta Crystallogr., Sect. B: Struct. Sci. 49, 435 (1993).

${ }^{15}$ J. D. Fitzgerald, R. L. Withers, A. M. Stewart, and A. Calka, Philos. Mag. B 58, 15 (1988).

${ }^{16}$ Z. Zhang and K. Urban, Philos. Mag. Lett. 60, 97 (1989).

${ }^{17}$ Z. Zhang and Y. Zhuang, Philos. Mag. Lett. 65, 203 (1992).

${ }^{18}$ Y. Yan, R. Wang, and J. Feng, Philos. Mag. Lett. 66, 197 (1992).

${ }^{19}$ Y. Yan and R. Wang, J. Mater. Res. 8, 286 (1993).

${ }^{20}$ Y. Yan and R. Wang, Philos. Mag. Lett. 67, 51 (1993).

${ }^{21}$ Z. Zhang, N. C. Li, and D. B. Williams, J. Appl. Phys. 73, 652 (1993).

${ }^{22}$ K. K. Fung, C. Y. Yang, Y. Q. Zhou, J. G. Zhao, W. S. Zhan, and B. G. Shen, Phys. Rev. Lett. 56, 2060 (1986).

${ }^{23}$ Y. Yan and R. Wang, Philos. Mag. Lett. 66, 253 (1992).

${ }^{24}$ Y. Yan, Z. Zhang, and R. Wang, Philos. Mag. Lett. 69, 123 (1994)

${ }^{25}$ D. Cherns and A. R. Preston, in Proceedings of the 11th Congress on Electron Microscopy, edited by T. Imura, S. Maruse and T. Suzuki (Japanese Society of Electron Microscopy, Kyoto, 1986), p. 721.

${ }^{26}$ R. Wang and Y. Chen, Mater. Sci. Forum 22-24, 409 (1987).

${ }^{27}$ J. Feng and R. Wang, Philos. Mag. A 69, 981 (1994).

${ }^{28}$ Y. Yan, R. Wang, J. Gui, M. Dai, and L. He, Philos. Mag. Lett. 65, 33 (1992).
${ }^{29}$ P. Schall, M. Feuerbacher and K. Urban (unpublished).

${ }^{30}$ W. Steurer and K. H. Kuo, Acta Crystallogr., Sect. B: Struct. Sci. 46, 703 (1990).

${ }^{31}$ W. Steurer, Acta Crystallogr., Sect. B: Struct. Sci. 45, 534 (1989).

${ }^{32}$ A. Cervellino, T. Haibach, and W. Steurer, Acta Crystallogr., Sect. B: Struct. Sci. 58, 8 (2002).

${ }^{33}$ K. N. Ishihara and A. Yamamoto, Acta Crystallogr., Sect. A: Found. Crystallogr. 44, 508 (1988).

${ }^{34}$ A. Pavlovitsch and M. Kléman, J. Phys. A 20, 687 (1987).

${ }^{35}$ S. Ritsch, C. Beeli, H.-U. Nissen, T. Gödecke, M. Scheffer, and R. Lück, Philos. Mag. Lett. 78, 67 (1998).

${ }^{36}$ M. Tanaka, M. Terauchi, and T. Kaneyama, Convergent Beam Electron Diffraction II (JEOL Ltd., Japan, 1988).

${ }^{37}$ M. Boudard, M. de Boossieu, C. Janot, G. Heger, C. Beeli, H.-U. Nissen, H. Vincent, R. Ibberson, M. Audier, and J. M. Dubois, J. Phys.: Condens. Matter 4, 10149 (1992).

${ }^{38}$ D. Hull and D. J. Bacon, Introduction to Dislocations (Pergamon Press, Oxford, 1984).

${ }^{39}$ R. Wang, M. Feuerbacher, M. Wollgarten, and K. Urban, Philos. Mag. A 77, 523 (1998).

${ }^{40}$ M. Wollgarten, R. Rosenfeld, M. Feuerbacher, Chr. Metzmacher, B. Baufeld, M. Bartsch, U. Messerschmidt, and K. Urban, in Proceedings of the 5th International Conference on Quasicrystals, Avignon 1995, edited by C. Janot and R. Mosseri (World Scientific, Singapore, 1995), p. 279.

${ }^{41}$ M. Wollgarten, Chr. Metzmacher, R. Rosenfeld, and M. Feuerbacher, Philos. Mag. A 76, 455 (1997).

${ }^{42}$ X.-F. Li, X.-Y. Duan, T.-Y. Fan, and Y.-F. Sun, J. Phys.: Condens. Matter 11, 703 (1999). 